\title{
An RNA thermometer
}

\author{
Gisela Storz \\ Cell Biology and Metabolism Branch, National Institute of Child Health and Human Development, National Institutes \\ of Health, Bethesda, Maryland 20892 USA
}

The expression of a conserved set of heat shock proteins is induced when cells grown at low temperatures are shifted to higher temperatures. Heat shock proteins are molecular chaperones or proteases that act to fold, translocate, or degrade proteins that appear to be misfolded or denatured upon heat shock. The heat shock response has been the focus of much research, and how the temperature signal is sensed and transduced to the biosynthetic machinery has been studied extensively.

The $\sigma^{32}(\mathrm{RpoH})$ alternative $\sigma$-factor, which is encoded by the rpoH gene, is a key regulator of the Escherichia coli heat shock response (for review, see Gross 1996; Missiakas et al. 1996). Upon a temperature shift from $30^{\circ} \mathrm{C}$ to $42^{\circ} \mathrm{C}, \sigma^{32}$ accumulates and directs RNA polymerase to the promoters of the heat shock genes (Fig. 1). Earlier studies showed that both increased synthesis and stability lead to the increased levels of $\sigma^{32}$. The activity of $\sigma^{32}$ and its association with RNA polymerase are also modulated by heat shock. A great deal has been learned about the increased stability of $\sigma^{32}$ in response to increased temperature. During normal growth the half-life of $\sigma^{32}$ is $\sim 1 \mathrm{~min}$; upon upshift the half-life is increased to $\sim 5$ min. Interestingly, the heat shock proteins DnaK, DnaJ, GrpE and HflB, whose expression is regulated by $\sigma^{32}$, function to destabilize $\sigma^{32}$. These proteins interact with $\sigma^{32}$, sequestering it away from RNA polymerase and targeting it for degradation. Misfolded proteins that accumulate after heat shock appear to titrate the DnaK, DnaJ, and GrpE chaperones and the HflB protease away from $\sigma^{32}$. Therefore, the pool of misfolded proteins is thought to be one measure of elevated temperature in the cell. Increased $\sigma^{32}$ synthesis was known to occur at the level of translation. However, although the E. coli heat shock response has been under investigation for many years, the thermometer signaling the need for increased translation was not known.

In this issue Morita et al. (1999) show that the secondary structure of the $r p o H$ mRNA itself is a thermosensor. These investigators present strong correlations between the expression of $r p o H-l a c Z$ fusions and the predicted and actual thermostability of the $r p o H$ mRNA secondary structure. In addition, rpoH-lacZ expression levels correlate with the formation of $r p o H$ mRNA-30S ribosometRNA $_{\mathrm{f}}{ }^{\mathrm{Met}}$ complexes. Thus, the melting of the rpoH mRNA secondary structure at high temperature leads to

E-MAIL storz@helix.nih.gov; FAX (301) 402-0078. ribosome binding and increased $\sigma^{32}$ synthesis. Here, I summarize the findings that led to this conclusion. I also contrast the $r p o H$ mRNA with other proposed RNA and protein thermometers.

\section{RNA thermometer of heat shock in E. coli}

T. Yura and colleagues (HSP Research Institute, Kyoto, Japan) first gained insight into the translational regulation of $r p o H$ by constructing an $r p o H-l a c Z$ translational fusion that carried $\sim 650 \mathrm{bp}$ of promoter sequence and most of the rpoH coding sequence. This fusion was induced strongly within $2 \mathrm{~min}$ after a shift from $30^{\circ} \mathrm{C}$ to $42^{\circ} \mathrm{C}$. Analyses of a series of $5^{\prime}$ and $3^{\prime}$ deletions of the rpoH-lacZ fusion indicated the presence of two regulatory elements (Fig. 1). One, $\sim 15$-nucleotide region (+6 to +20 ), immediately downstream of the AUG initiation codon and denoted region A, was required for high-level expression of the rpoH-lac Z fusion. A second, 97 nucleotide region $(+112$ to +208$)$, internal to the $r p o H$ coding sequence and denoted region $\mathrm{B}$, was required for thermal regulation. Computer analysis predicted that the $5^{\prime}$ region of the $r p o H$ mRNA $(-19$ to +247$)$ might form a complex secondary structure with the region $\mathrm{A}$ and the initiation codon base-pairing to parts of region $\mathrm{B}$ (Fig. 1). This proposed structure was predicted to inhibit ribosome binding to the rpoH message.

Support for the inhibitory rpoH mRNA secondary structure has come from several lines of evidence. First, the levels of expression detected from $\mathrm{rpoH}-\mathrm{lac} \mathrm{Z}$ fusions carrying base substitutions or internal deletions were consistent with the secondary structure preventing $r p o H$ mRNA translation (Yuzawa et al. 1993). For example, mutations that were predicted to weaken the secondary structure led to constitutive expression. Thermoregulation could be restored by compensatory mutations. In addition, the predicted secondary structure is conserved in Citrobacter freundii, Enterobacter cloacae, Serratia marcescens, Proteus mirabilis, and Pseudomonas aeruginosa rpoH messages (Nakahigashi et al. 1995), and the $S$. marcescens and $P$. aeruginosa rpoH genes expressed in $E$. coli show the same temperature regulation as the $E$. coli clone (Nakahigashi et al. 1998). Furthermore, the results of recent chemical and enzymatic probing of the rpoH mRNA secondary structure are completely consistent with the proposed model (Morita et al. 1999a). Structural probing also showed that RNAs with muta- 


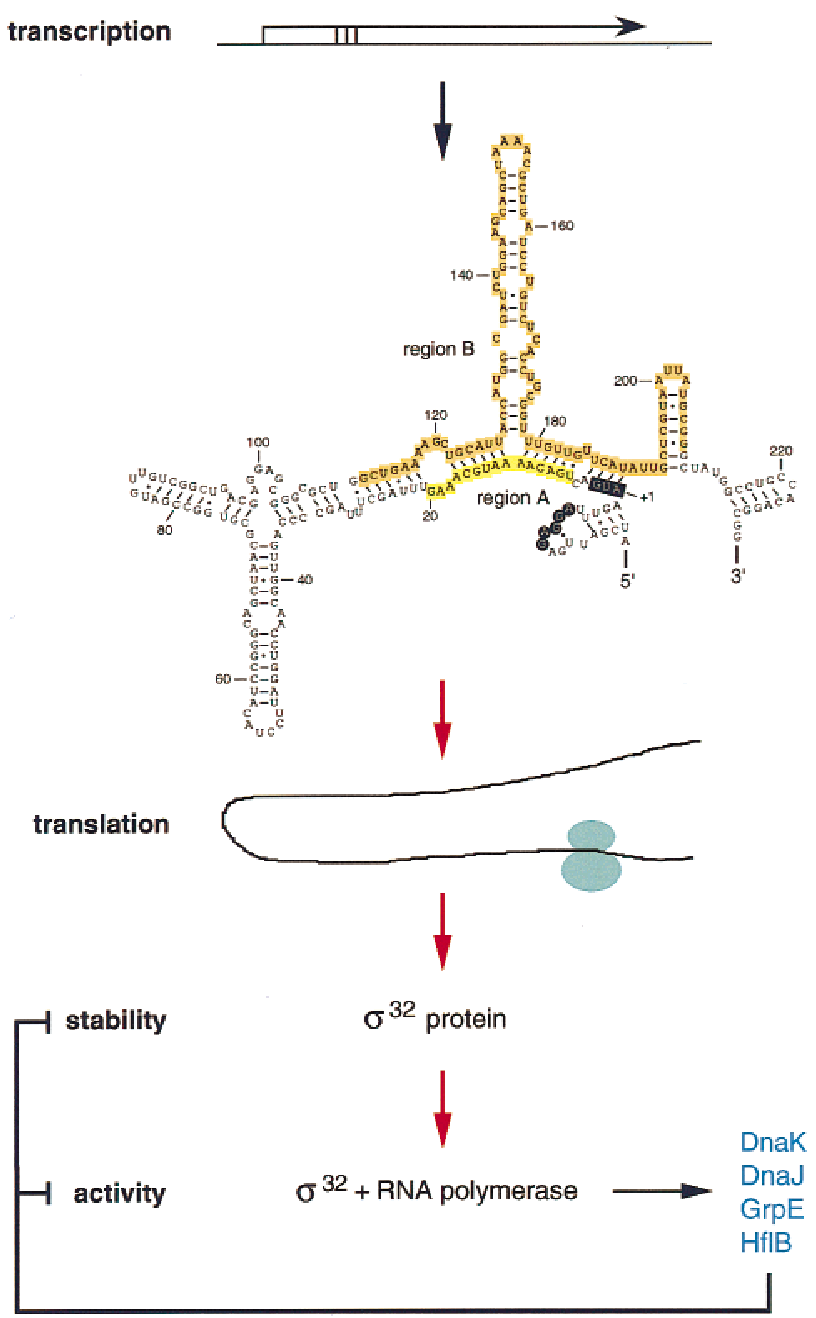

Figure 1. The expression of $\sigma^{32}$ is regulated at the levels of transcription, translation, and protein stability and $\sigma^{32}$ activity. Although rpoH transcription is not induced by $30^{\circ} \mathrm{C}$ to $42^{\circ} \mathrm{C}$ temperature shifts, $\mathrm{rpoH}$ transcription is controlled from four different promoters. One of these promoters is recognized by the $\sigma^{\mathrm{E}}$-RNA polymerase, which acts to induce $r p o H$ expression at extreme temperatures $>50^{\circ} \mathrm{C}$. $r p o H$ mRNA translation, $\sigma^{32}$ stability, and $\sigma^{32}$ activity are all induced by temperature shifts from $30^{\circ} \mathrm{C}$ to $42^{\circ} \mathrm{C}$ (red arrows). As shown by Morita et al. (1999b), the rpoH mRNA secondary structure itself is a thermosensor. At lower temperatures, the $r p o H$ mRNA is folded into a secondary structure that occludes the ribosome binding site (circled) and the initation codon (boxed). Upon heat shock, this structure is unfolded allowing ribosome binding (in green) and increased $\sigma^{32}$ synthesis.

tions predicted to disrupt base-pairing have altered secondary structures. In the course of this recent study, Morita et al. (1999a) constructed a minimal rpoH-lacZ fusion, containing only 97 nucleotides of the rpoH coding region, which exhibited normal thermoregulation. In this construct some of the stem loops are shortened, but the pairing between regions $\mathrm{A}$ and $\mathrm{B}$ is maintained.

In this issue Morita et al. (1999b) used the minimal rpoH-lacZ construct to further examine the thermoregulation of $r p o H$ translation. These workers show that mu- tations predicted to decrease stability give rise to increased expression and mutations predicted to increase stability give rise to decreased expression. Subsequently, they used circular dichroism (CD) to directly measure the temperature-melting profiles of different RNAs. The measured thermostability correlated with the levels of expression observed from the fusion constructs. For example, a mutant RNA carrying a $\mathrm{C} \rightarrow \mathrm{A}$ substitution at position $+15(15 \mathrm{~A})$, which shows increased expression at $30^{\circ} \mathrm{C}$ and therefore reduced thermoinduction, was denatured at lower temperature than the control RNA. A mutant RNA carrying 15A and a compensatory mutation of position +124 (15A-124U) showed nearly wild-type thermoregulation and wild-type thermostability.

The translation initiation region of the rpoH mRNA is paired with region B in the secondary structure. Therefore, T. Yura and colleagues proposed that ribosome entry was occluded under nonstress conditions. Stress conditions would lead to disruption of the secondary structure, ribosome binding, and enhanced translation. To directly test this model, Morita et al. (1999b) carried out toeprinting assays (also denoted primer-extension inhibition assays). In these in vitro experiments, a primer is hybridized downstream of the ribosome binding site and extended with reverse transcriptase. Binding of $30 \mathrm{~S}$ ribosome subunits to the mRNA in the presence of $\mathrm{tRNA}_{\mathrm{f}}^{\text {Met }}$ blocks reverse transcriptase elongation, resulting in a 'toeprint' $\sim 15$ nucleotides from the initiation codon. In the absence of $30 \mathrm{~S}$ binding this toeprint is not observed. For the wild-type $r p o H$ RNA, no toeprint was observed at $30^{\circ} \mathrm{C}$, indicating that formation of the ternary complex is prevented at this temperature. In contrast, a toeprint appeared within $5 \mathrm{~min}$ of incubation at $42^{\circ} \mathrm{C}$, demonstrating that $30 \mathrm{~S}$ binding does occur at the higher temperature. These researchers also carried out toeprint assays for a subset of the mutant RNAs. Again, the extent of ribosome binding correlated with the stability of the RNA secondary structure. The 15A consitutive mutant showed a toeprint at all temperatures, whereas the toeprint profile of the thermal regulated 15A-124U mutant at different temperatures was closer to that of the wildtype RNA.

Together, these results clearly establish that translation of $r p o H$ is regulated by the intrinsic stability of the rpoH mRNA secondary structure. It had long been suggested that a hypothetical factor would be required for the thermoregulation of $r p o H$ translation. The results of the toeprint assays, which were carried out in the absence of any additional factors, show that rpoH structure alone can control $\sigma^{32}$ expression. These experiments have provided an answer to the long-standing puzzle of the nature of the cellular thermometer that gauges the need for increased $\sigma^{32}$ synthesis. However, some interesting questions remain. The extensive secondary structure, containing four stem-loops is quite conserved between E. coli and other bacteria, although Morita et al. (1999a) found that a truncated derivative still exhibits normal thermoregulation in their assays. Do the conserved stem-loops have additional roles? What structural features determine the set point of the rpoH mRNA 
thermometer? Is the activation reversed by simple refolding of the RNA structure? Do any other RNA or protein factors contribute to the regulation?

\section{Other RNA thermometers}

Correlations between levels of expression, temperature, and RNA secondary structure have been reported for a few other RNAs. One example is the lcrF mRNA of Yersinia pestis (Hoe and Goguen 1993). lcrF encodes a transcription factor responsible for inducing the expression of plasmid-encoded virulence genes in response to temperature. A comparison of the amount of LcrF protein produced per unit of message at $26^{\circ} \mathrm{C}$ compared to $37^{\circ} \mathrm{C}$ indicated that the efficiency of $1 c r F$ mRNA translation increased with temperature. In predictions of the $1 c r F$ mRNA secondary structure, the $1 c r F$ ribosome binding site is sequestered in a stem-loop. These results led Hoe and Goguen (1993) to propose that the decreased stability of the stem-loop with increasing temperature leads to increased efficiency of translation initiation. This model needs to be tested but is very similar to the mechanism elucidated for $r p o H$. A second example is the mRNA encoding the $c$ III gene of bacteriophage $\lambda$ (Altuvia et al. 1989). In vitro experiments showed that the cIII mRNA can exist in two conformations. High temperatures $\left(45^{\circ} \mathrm{C}\right)$ and mutations that increase $c \mathrm{III}$ expression promoted the formation of one structure in which ribosome binding is efficient. By contrast, low temperatures $\left(37^{\circ} \mathrm{C}\right)$ and mutations that reduce $\mathrm{cIII}$ expression promoted the formation of a second structure in which the translation region is occluded and ribosome binding is reduced. It is likely that other RNA species that possess different secondary structures at different temperatures can function as physiological sensors of either high or low temperature.

\section{Protein thermometers}

The activities of two DNA-binding proteins, Salmonella typhimurium TlpA and Drosophila HSF, are also directly sensitive to temperature (Hurme et al. 1997; Zhong et al. 1998). TlpA, which is encoded on a virulence plasmid, is a transcriptional repressor of its own synthesis. Expression of a $t l p A-l a c Z$ transcriptional fusion is elevated 13.2 -fold between $37^{\circ} \mathrm{C}$ and $45^{\circ} \mathrm{C}$, and this regulation is dependent on TlpA. Gel mobility shift assays showed that the DNA-binding activity of purified TlpA is sensitive to temperature with less binding observed at $43^{\circ} \mathrm{C}$ than at $22^{\circ} \mathrm{C}$. Thus, Hurme et al. (1997) propose the temperature shift that occurs upon entry of Salmonella into a host organism would lead to the derepression of tlpA and other as-yet-unidentified TlpA target genes. Drosophila HSF activates the expression of target heat shock genes in response to elevated temperatures. The transcription factor is normally present in a latent, monomeric form that is unable to bind DNA. Initial activation of HSF entails the conversion of monomers to homotrimers that bind to DNA with high affin- ity. Using gel filtration chromatography and equilibrium sedimentation, Zhong et al. (1998) recently showed that the trimerization and DNA binding of purified HSF can be directly induced by heat shock temperatures in vitro such that a higher percentage of trimers is observed at $40^{\circ} \mathrm{C}$ compared to $20^{\circ} \mathrm{C}$.

It is intriguing that coiled-coil $\alpha$-helices are implicated as part of the temperature-sensitive switch in both TlpA and HSF. TlpA contains a long coiled-coil domain that can switch between unfolded (monomer) and folded (coiled-coil, oligomer) states. Only the folded form of TlpA can act as a repressor. Hurme et al. (1997) suggest that high temperatures bring about the unfolding of the coiled-coil domain leading to the formation of nonfunctional monomers and the derepression of $\operatorname{tlp} A$ expression. The HSF trimerization domain encompasses several hydrophobic heptad repeats that are likely to assume a three-stranded coiled-coil structure in the HSF trimer. This three-stranded structure is thought to be precluded from forming in the monomer due to formation of an intramolecular coiled-coil structure. Thus, Zhong et al. (1998) suggest that activation of HSF may occur by a heat-induced conformational change that unmasks the trimerization domain in the monomer allowing trimer assembly. Further studies need to be carried out to elucidate the structures of TlpA and HSF at low and high temperatures. Whether coiled-coil $\alpha$-helices are generally utilized as thermosensing domains in other proteins remains to be seen.

\section{More thermometers?}

Organisms are exposed to changes in temperature under a variety of conditions. In many environments, the temperature fluctuates substantially between day and night. Pathogens often encounter elevated temperatures when they enter the host organisms. Given the universal need to sense and respond to both increased and decreased environmental temperature, it is likely that many other thermosensors remain be discovered. It will be interesting to compare the sensing mechanisms of these thermometers with the RNAs and proteins described above.

\section{Acknowledgments}

I thank Susan Gottesman, David Wassarman, Karen Wassarman, and Carl Wu for helpful comments on the manuscript and Aixia Zhang for her help with Figure 1.

\section{References}

Altuvia, S., D. Kornitzer, D. Teff, and A.B. Oppenheim. 1989. Alternative mRNA structures of the $c$ III gene of bacteriophage $\lambda$ determine the rate of its translation initiation. $J$. Mol. Biol. 210: 265-280.

Gross, C.A. 1996. Function and regulation of the heat shock proteins. In Escherichia coli and Salmonella: Cellular and molecular biology (ed. F.C. Neidhardt, R. Curtiss, III, J.L. Ingraham, E.C.C. Lin, K.B. Low, B. Magasanik, W.S. Reznikoff, M. Riley, M. Schaechter, and H.E. Umbarger), pp. 13821399. ASM Press, Washington, D.C. 
Hoe, N.P. and J.D. Goguen. 1993. Temperature sensing in Yersinia pestis: Translation of the LcrF activator protein is thermally regulated. J. Bacteriol. 175: 7901-7909.

Hurme, R., K.D. Berndt, S.J. Normark, and M. Rhen. 1997. A proteinaceous gene regulatory thermometer in Salmonella. Cell 90: 55-64.

Missiakas, D., S. Raina, and C. Georgopoulos. 1996. Heat shock regulation. In Regulation of gene expression in Escherichia coli (ed. E.C.C. Lin and A.S. Lynch), pp. 481-501. R.G. Landes Company, Austin, TX.

Morita, M., M. Kanemori, H. Yanagi, and T. Yura. 1999a. Heatinduced synthesis of $\sigma^{32}$ in Escherichia coli: Structural and functional dissection of $r p o H$ mRNA secondary structure. $J$. Bacteriol. 181: 401-410.

Morita, M.T., Y. Tanaka, T.S. Kodama, Y. Kyogoku, H. Yanagi, and T. Yura. 1999b. Translational induction of heat shock transcription factor $\sigma^{32}$ : Evidence for a built-in RNA thermosensor. Genes \& Dev. (this issue).

Nagai, H., H. Yuzawa, and T. Yura. 1991. Interplay of two cisacting mRNA regions in translational control of $\sigma^{32}$ synthesis during the heat shock response of Escherichia coli. Proc. Natl. Acad. Sci. 88: 10515-10519.

Nakahigashi, K., H. Yanagi, and T. Yura. 1995. Isolation and sequence analysis of $r p o H$ genes encoding $\sigma^{32}$ homologs from gram negative bacteria: conserved mRNA and protein segments for heat shock regulation. Nucleic Acids Res. 23: 4383-4390.

. 1998. Regulatory conservation and divergence of $\sigma^{32}$ homologs from gram-negative bacteria: Serratia marcescens, Proteus mirabilis, Pseudomonas aeruginosa, and Agrobacterium tumefaciens. J. Bacteriol. 180: 2402-2408.

Yuzawa, H., H. Nagai, H. Mori, and T. Yura. 1993. Heat induction of $\sigma^{32}$ synthesis mediated by mRNA secondary structure: a primary step of the heat shock response in Escherichia coli. Nucleic Acids Res. 21: 5449-5455.

Zhong, M., A. Orosz, and C. Wu. 1998. Direct sensing of heat and oxidation by Drosophila heat shock transcription factor. Mol. Cell 2: 101-108. 


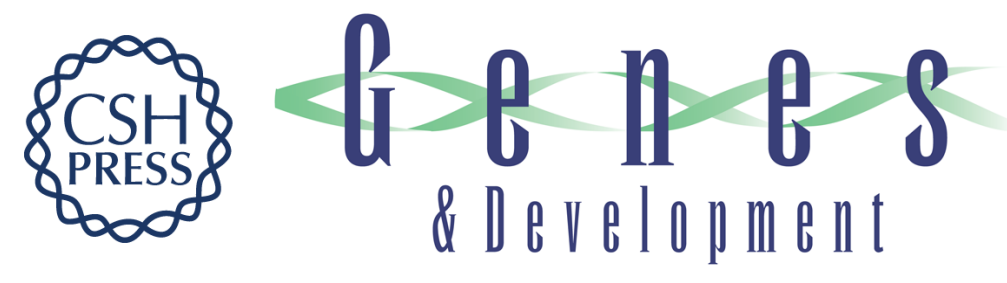

\section{An RNA thermometer}

Gisela Storz

Genes Dev. 1999, 13:

References This article cites 8 articles, 4 of which can be accessed free at: http://genesdev.cshlp.org/content/13/6/633.full.html\#ref-list-1

License

Email Alerting

Receive free email alerts when new articles cite this article - sign up in the box at the top Service right corner of the article or click here.

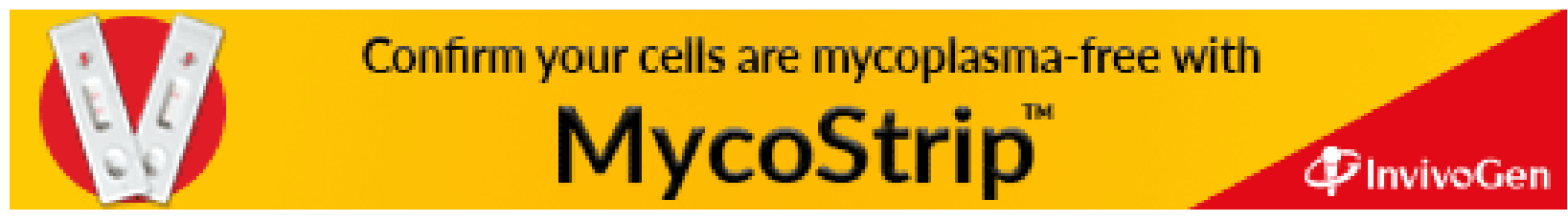

